\title{
Interaction of Inferior Temporal Cortex with Frontal Cortex and Basal Forebrain: Double Dissociation in Strategy Implementation and Associative Learning
}

\author{
David Gaffan, ${ }^{1}$ Alexander Easton, ${ }^{2}$ and Amanda Parker ${ }^{2}$ \\ ${ }^{1}$ Department of Experimental Psychology, Oxford University, Oxford OX1 3UD, United Kingdom, and 2School of \\ Psychology, Nottingham University, Nottingham NG7 2RD, United Kingdom
}

Macaque monkeys learned a strategy task in which two groups of visual objects needed to be treated differently, one with persistent and one with sporadic object choices, to obtain food rewards. After preoperative training, they were divided into two surgical groups of three monkeys each. One group received crossed unilateral removals of frontal cortex and inferior temporal cortex $(\mathrm{IT} \times \mathrm{FC})$ and were severely impaired in performing the strategy task. The other group received bilateral transection of anterior temporal stem, amygdala, and fornix (TS+AM+FX) and were unimpaired in performing the strategy task. Subsequently the same animals were tested in visual object-reward association learning. Here, confirming previous results, group IT $\times$ FC was unimpaired, whereas group $\mathrm{TS}+\mathrm{AM}+\mathrm{FX}$ was severely impaired. The results show that the amnesic effects of

A severe memory impairment can be produced in the monkey by surgically interrupting the axons in the white matter of the medial temporal lobe (Gaffan et al., 2001; Maclean et al., 2001). These axons include projections that rise from the basal forebrain and midbrain to innervate widespread areas of lateral as well as medial temporal cortex. The ascending axons reach the temporal cortex through three routes (Selden et al., 1998): in the fornix-fimbria, in fibers of passage through the amygdala, and in the anterior temporal stem, which is the white matter surrounding the amygdala dorsally and laterally. All three routes are interrupted by the surgical excisions in H. M. [for the anterior temporal stem damage, see Corkin et al. (1997), their Fig. $2 H-J]$. A severe memory impairment results in the monkey only when all three routes (but not any subset of only two routes) are interrupted (Gaffan et al., 2001). The same functional effect can be produced by a very different surgical manipulation in the monkey, namely disconnection by crossed unilateral lesions of basal forebrain in one hemisphere and temporal cortex in the other (Easton and Gaffan, 2000, 2001; Easton et al., 2001, 2002). The similar effects of these two very different surgical manipulations in the monkey, and the similarity of each of them to the effect of a third different surgical procedure in the human brain (Corkin et al., 1997), support the idea that their common feature, namely the subcortical disconnection of temporal cortex, is the explanation of dense amnesia after temporal lobe lesions, both in human patients and in monkeys.

Received March 6, 2002; revised May 13, 2002; accepted May 24, 2002.

This research was supported by a grant from the Medical Research Council UK.

Correspondence should be addressed to Dr. D. Gaffan, Department of Experimental Psychology, Oxford University, South Parks Road, Oxford OX1 3UD, UK. E-mail: gaffan@psy.ox.ac.uk.

Copyright (ㄷ) 2002 Society for Neuroscience $\quad 0270-6474 / 02 / 227288-09 \$ 15.00 / 0$
$\mathrm{TS}+\mathrm{AM}+\mathrm{FX}$ cannot be generally attributed to the partial temporal-frontal disconnection that this lesion creates, and therefore support the hypothesis that the amnesic effects of this lesion are caused primarily by the disconnection of temporal cortex from ascending inputs from the basal forebrain. The results also show that temporal-frontal interaction in strategy implementation does not require those routes of temporalfrontal interaction that are interrupted in $T S+A M+F X$, and therefore support the hypothesis that projections to other posterior cortical areas allow temporal and frontal cortex to interact with each other by multisynaptic corticocortical routes in strategy implementation.

Key words: amnesia; frontal cortex; visual memory; conditional learning; executive function; cortical plasticity

Surgical section through the anterior temporal stem, amygdala, and fornix also interrupts many potential routes of interaction between temporal and frontal cortex, however, and it is possible that this alone, rather than the subcortical disconnection of temporal cortex, is sufficient to explain much of the amnesic effect of sectioning anterior temporal stem, amygdala, and fornix. If this were true, however, similar amnesic effects would be obtained by any other surgical manipulation that disconnected frontal-temporal interaction. Because the pathways of interaction between frontal and temporal cortex are primarily ipsilateral, frontaltemporal interaction can be disconnected by making crossed unilateral ablations of the temporal cortex in one hemisphere and the frontal cortex in the opposite hemisphere. This combination of ablations severely impaired monkeys' ability to perform conditional discriminations that they learned preoperatively (Gaffan and Harrison, 1988, 1991; Parker and Gaffan, 1998). The main purpose of the present experiment, therefore, was to compare directly the effects of these two surgical manipulations in two groups of monkeys, one (group $\mathrm{TS}+\mathrm{AM}+\mathrm{FX}$ ) receiving bilateral section of anterior temporal stem, amygdala, and fornix, and the other (group IT $\times$ FC) receiving crossed unilateral ablations of the inferior temporal cortex in one hemisphere and the frontal cortex in the opposite hemisphere. The animals were tested in two tasks: the postoperative implementation of a preoperatively acquired conditional strategy task and the postoperative new learning of object-reward associations.

\section{MATERIALS AND METHODS}

Subjects. We used six experimentally naive macaque monkeys, S1-S6. S1 and S2 were Cynomolgus (Macaca fascicularis), and the remaining animals were Rhesus $(M$. mulatta $)$. S6 was female, and the other animals 


\begin{tabular}{llll}
\hline \multicolumn{3}{l}{ Table 1. Surgical operations for each monkey } \\
Group & Monkey & First operation & Second operation \\
\hline IT $\times$ FC & S1 & FC right & IT left \\
& S2 & IT right & FC left \\
& S3 & FC left & IT right \\
TS + AM + FX & S4 & TS + AM bilateral & FX bilateral \\
& S5 & TS + AM bilateral & FX bilateral \\
& S6 & TS + AM bilateral & FX bilateral
\end{tabular}

$\overline{\text { FC, Unilateral ablation of frontal cortex; IT, unilateral ablation of inferior temporal }}$ cortex; TS+AM, section of anterior temporal stem and amygdala; FX, fornix section.
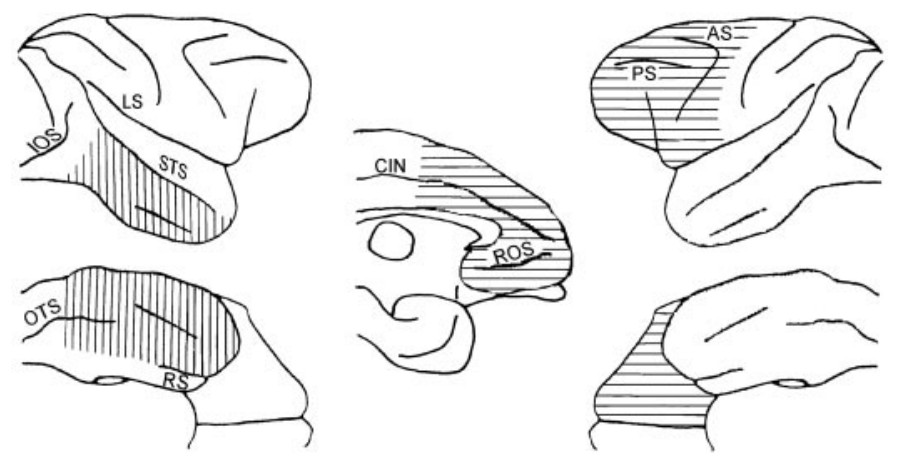

Figure 1. The lesion in group IT $\times$ FC (monkeys S1-S3). On the left of the figure the unilateral inferior temporal ablation is shown in vertical hatching in lateral (top) and basal (bottom) views of the right hemisphere. On the right of the figure the unilateral frontal ablation is shown in horizontal hatching in lateral (top) and basal (bottom) views of the left hemisphere, and in the center the unilateral frontal ablation is shown in a medial view of the left hemisphere. $A S$, Arcuate sulcus; $C I N$, cingulate sulcus; IOS, inferior occipital sulcus; $L S$, lateral sulcus; OTS, occipitotemporal sulcus; $P S$, principal sulcus; $R O S$, rostral sulcus; $R S$, rhinal sulcus; STS, superior temporal sulcus.

were male. At the time of their first surgery they weighed between 3.7 and $5.6 \mathrm{~kg}$. After the completion of preoperative training the animals were divided into two groups for surgery to equalize the preoperative ability of the two groups. Animals S1-S3 formed group IT $\times$ FC, which in two stages of surgery received crossed unilateral ablations of frontal and inferior temporal cortex. Animals S4-S6 formed group $\mathrm{TS}+\mathrm{AM}+\mathrm{FX}$, which in two stages of surgery received bilateral transection of the anterior temporal stem, amygdala, and fornix.

Surgery. The order in which the animals received surgical operations is shown in Table 1 . The operations were performed under aseptic conditions. The monkeys were anesthetized throughout surgery with barbiturate (thiopentone sodium) administered through an intravenous cannula. The ablations were made by aspiration under visual guidance with the aid of an operating microscope. At the end of the operation the tissue was closed in layers. After each operation, the animals rested for 10-14 d before beginning postoperative behavioral testing. The surgical methods described below are identical to those in the other recent experiments from our laboratory that have investigated the effects of these ablations.

Inferior temporal cortex ablation. This operation was performed unilaterally in group IT $\times$ FC (Table 1$)$. After coronal incision of the skin and galea, the arch of the zygoma was removed, and the temporal muscle was detached from the cranium and retracted. A bone flap was raised and extended with a rongeur over the area of the ablation, and the dura mater was incised and retracted. Pia mater was cauterized to control bleeding, and the cortical gray matter was removed by aspiration. The extent of the ablation is shown in Figure 1. The ablation extended from the fundus of the superior temporal sulcus to the fundus of the rhinal sulcus and posteriorly included both banks of the anterior part of the occipitotemporal sulcus. The posterior limit of the ablation was a line drawn perpendicular to the superior temporal sulcus, $5 \mathrm{~mm}$ anterior to the inferior occipital sulcus. The anterior limit of the ablation was the anterior tip of the superior temporal sulcus and a line drawn round the pole from that tip to the rhinal sulcus. Within these limits all the cortex was removed, including both banks of the anterior and posterior middle temporal sulci.

Frontal cortex ablation. This operation was performed unilaterally in group IT $\times$ FC $($ Table 1$)$. After sagittal incision of the skin and galea, a bone flap was raised over the area of the intended ablation, and the dura mater was incised. The intention was to remove the entire frontal cortex in one hemisphere except for primary motor cortex. Pia mater was cauterized to control bleeding, and the cortical gray matter was removed by aspiration. The extent of the removal is shown in Figure 1. The cortical gray matter was removed from the surface of the brain and from sulci within the boundary shown, and the underlying white matter surrounding the corpus striatum was left intact.

Temporal stem and amygdala section. This operation was performed bilaterally in group TS $+\mathrm{AM}+\mathrm{FX}$ (Table 1). After coronal incision of the skin and galea, the temporal muscle was detached from the cranium and retracted. The zygomatic arch was left intact. The frontal and temporal bone overlying the anterior part of the lateral sulcus was removed with a rongeur. The dura mater was incised, and the pia mater along the lip of the lower bank of the lateral sulcus was cauterized from the temporal pole to the level of the central sulcus. The cortex within the lower bank of the lateral sulcus was removed, exposing the pia mater in the lower limb of the insula. The white matter of the anterior temporal stem, adjacent to the foot of the insula, was sectioned by aspiration. The gray matter of the dorsolateral amygdala appeared when the section through the anterior temporal stem was completed. Using aspiration and cautery, the section was then extended through the amygdala until the pia mater on the medial wall of the temporal lobe was encountered. The aim was to section at a dorsal level in the amygdala to leave the perirhinal cortex intact. The section through anterior temporal stem and amygdala was then extended posteriorly to reveal the lateral ventricle and the anteriordorsal surface of the hippocampus. The hippocampus was left intact.

Fornix section. This operation was performed bilaterally in group $\mathrm{TS}+\mathrm{AM}+\mathrm{FX}$ (Table 1). After sagittal incision of the skin and galea, a bone flap was raised over the midline, and the dura mater was incised over one hemisphere in a crescent shape and retracted to the sagittal sinus. Veins running from the cortex into the sagittal sinus and dura mater were cauterized and cut. The exposed hemisphere was retracted with a brain spoon, and the corpus callosum was exposed. The corpus callosum was sectioned with a glass aspirator in the midline, in the region of the anterior thalamus and interventricular foramen, exposing the fornix, and the fornix was sectioned with cautery and aspiration. The flap was replaced, and the wound was closed in layers.

Histology. At the conclusion of the behavioral testing, all of the animals were deeply anesthetized and transcardially perfused with physiological saline followed by $10 \%$ formalin. The brains were blocked in the coronal stereotaxic plane, extracted from the cranium, and cut on a freezing microtome in $50 \mu \mathrm{m}$ sections in the coronal plane. The sections were stained with cresyl violet, mounted on slides, and coverslipped.

All three animals in group IT $\times$ FC $($ S1-S3) had ablations that corresponded closely to the intended removals shown in Figure 1. The ablations included the cortical tissue in the sulci within the intended removal, as well as the cortex on the surface of the hemisphere. The ablations in the animals of group $\mathrm{TS}+\mathrm{AM}+\mathrm{FX}(\mathrm{S} 4-\mathrm{S} 6)$ are shown in Figure 2. These drawings were made in the same way as the drawings of this ablation in the initial report of the behavioral effects of this ablation in a large series of monkeys (Gaffan et al., 2001). It can be seen that the transection through temporal stem, amygdala, and fornix was complete bilaterally in all three animals. In animals S5 and S6, the cortical damage to the anterior lateral temporal lobe was limited to the superior temporal gyrus, as intended, leaving intact the visual association cortex of the middle and inferior temporal gyri, that is, the cortex between the fundus of the superior temporal sulcus and the fundus of the rhinal sulcus. In animal S4, there was unintended damage in one hemisphere to the perirhinal cortex, lateral to the rhinal sulcus. Posteriorly in all animals the transection entered the lateral ventricle and spared the hippocampus, as intended.

Apparatus. The monkey was brought to the training apparatus in a wheeled transport cage (floor area $600 \mathrm{~mm}$ wide and $500 \mathrm{~mm}$ deep), which was then fixed to the front of the apparatus. The monkey could reach out through bars at the front of the transport cage to touch a touch-sensitive color monitor screen that was $150 \mathrm{~mm}$ from the front of the cage. The screen was $380 \mathrm{~mm}$ wide and $280 \mathrm{~mm}$ high. A closed-circuit television system allowed the experimenters to watch the monkey from another room, and the room with the monkey and apparatus contained no other monkeys or people during the test sessions. Small food rewards 
S4

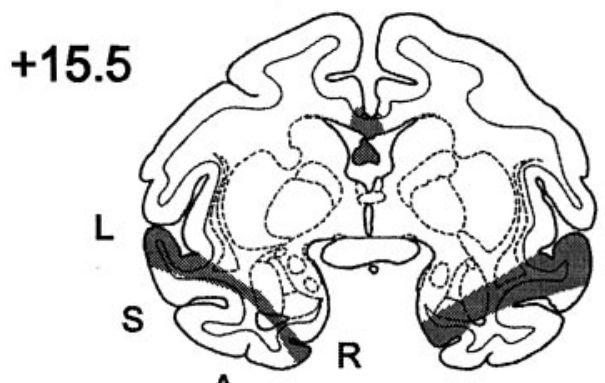

A

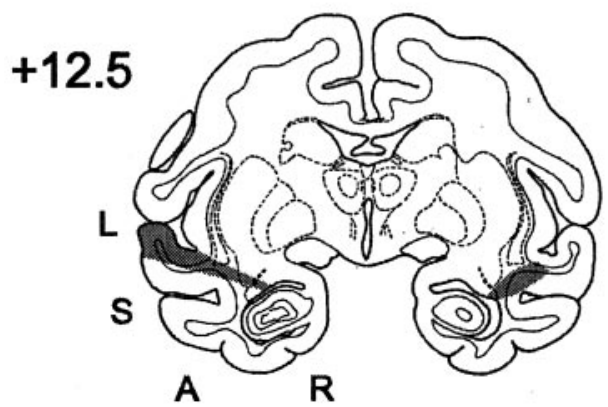

S5
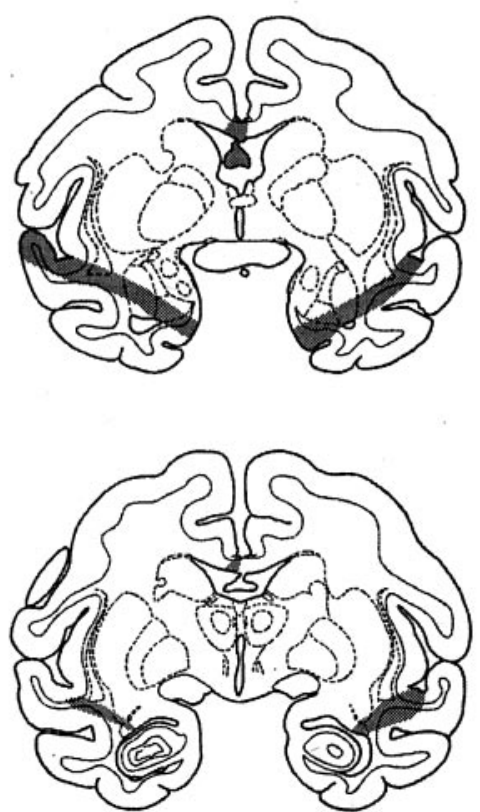

S6
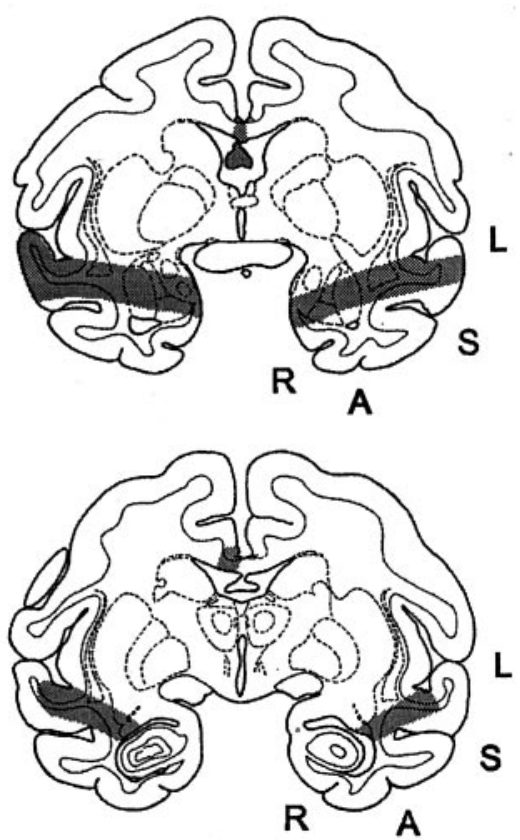

Figure 2. The lesion in Group TS +AM+FX (monkeys S4-S6). For each of the three monkeys the lesion is shown on drawings of coronal sections of a standard rhesus monkey brain at two levels, $15.5 \mathrm{~mm}$ anterior to the auditory meatus (top) and $12.5 \mathrm{~mm}$ anterior to it (bottom). L, Lateral sulcus; $S$, superior temporal sulcus; $A$, anterior middle temporal sulcus; $R$, rhinal sulcus.

(pellets specially formulated for monkeys, $190 \mathrm{mg}$ ) were delivered into a hopper placed centrally underneath the monitor screen. A single large food reward was delivered at the end of each training session by opening a box that was set to one side of the centrally placed hopper. The box contained peanuts, raisins, proprietary monkey food, fruit, and seeds. The amount of this large reward was adjusted for individual animals to avoid obesity. Opening of the box with the large food reward, like all other aspects of the events and the experimental contingencies during any session of training, was under computer control. The purpose of delivering the large reward was to give the animals an additional incentive to perform the tasks. The small and large rewards dispensed in the training apparatus provided almost the whole daily diet of the monkeys on days with a test session.

Stimulus material. The visual objects were colored monograms, constructed from a set of 574 typographic characters that were all different from each other. To make one object, two characters were chosen randomly from the set, each was independently given a random color, and they were abutted side by side, just touching each other, to form a monogram. The characters were chosen with the constraint that no character could appear in more than one object for any monkey. The colors were chosen with a brightness constraint that varied with the size of the character, dimmer colors being permitted for characters of larger area. The objects were $\sim 35 \mathrm{~mm}$ high and $45 \mathrm{~mm}$ wide on average. For each individual monkey, the objects to be learned, in both of the experimental tasks, were unique to that monkey. Thus, any variance attributable to differences between individual sets of objects is controlled in the same way as variance attributable to differences between individual monkeys, variance between object sets being confounded with variance between monkeys.

Procedure: preliminary training. Animals were initially trained to touch objects on the touch screen by autoshaping procedures similar to those described previously in detail (Gaffan et al., 1984). They then learned some object-reward associations, similar to those in the postoperative test of object-reward associative learning described below, before beginning the preoperative training schedule described below. Stimulus objects that were used in this preliminary training did not reappear in the subsequent tasks.
Procedure: preoperative acquisition of the strategy task. The strategy task was similar to conventional conditional learning in that different choices were appropriate on different trials but similar to human strategy implementation in that the strategy currently being followed by the subject, rather than any discrete external cue, dictated which choices were appropriate on any trial. Two means of obtaining food reward, "persistent" and "sporadic" pursuit of the reward, were each implemented by the choice of objects from one or the other of two arbitrarily defined classes of visual objects, one class for the persistent strategy and the other for the sporadic strategy.

In the main task, after the introductory training described below, there were four pairs of objects (described under Stimulus material). The pairs were used once each in random order in a block of four trials and then again in a new random order in the next block of four trials and so on until the last trial in a session. Within each pair of objects, one of the objects, at random, was designated a "persistence" object (type P) and the other was designated a "sporadic" object (type S). On any trial in the task, a pair of objects was presented side by side on the screen with its left-right position determined at random, and the animal chose one object by touching it. The animal had to learn by trial and error which object was of which type. Food rewards (described under Apparatus) were dispensed according to the schedule described below. Whichever choice was made, both objects disappeared from the screen and an intertrial interval of $5 \mathrm{sec}$ began, during which any touch to the screen reset the interval.

The reward schedule was based on the idea that P-type objects rewarded only a persistent attempt to gain reward from them, whereas S-type objects rewarded only a sporadic attempt to gain reward from them. A persistence-type reward was obtained if and only if four successive choices of P-type objects were made on four successive trials. Choices of type $\mathrm{S}$ were rewarded only if at least four successive trials had previously passed without any $\mathrm{S}$ choice. Various overall strategies can be adopted, of which the simplest is just to make persistent choices all the time; this strategy produces one reward in every four trials. The most efficient overall strategy is to pursue $\mathrm{P}$ for four trials then switch to $\mathrm{S}$ for just one trial then switch back to P for a further four trials, and so on. This efficient strategy was shown by all monkeys at the end of preoper- 
Table 2. Preoperative acquisition of the strategy task

Rewards to criterion with pairs of objects

\begin{tabular}{|c|c|c|c|c|c|c|c|}
\hline \multirow[b]{2}{*}{ Monkey } & \\
\hline & 1 & 2 & 3 & 4 & All & Total & First ratio \\
\hline S1 & 360 & 840 & 400 & 350 & 400 & 2350 & 2.98 \\
\hline $\mathrm{S} 2$ & 420 & 180 & 120 & 240 & 840 & 1800 & 3.17 \\
\hline S3 & 800 & 640 & 300 & 220 & 560 & 2520 & 3.03 \\
\hline $\mathrm{IT} \times \mathrm{FC}$ mean & 527 & 553 & 273 & 270 & 600 & 2223 & 3.03 \\
\hline $\mathrm{S} 4$ & 225 & 125 & 565 & 70 & 280 & 1265 & 2.93 \\
\hline S5 & 1705 & 210 & 390 & 160 & 930 & 3395 & 3.04 \\
\hline S6 & 450 & 600 & 270 & 100 & 640 & 2060 & 3.55 \\
\hline $\mathrm{TS}+\mathrm{AM}+\mathrm{FX}$ mean & 793 & 312 & 408 & 110 & 617 & 2240 & 3.03 \\
\hline
\end{tabular}

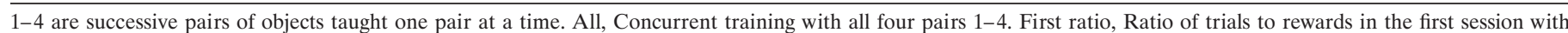
all four pairs concurrently.

ative training and is illustrated in Figures 4 and 5. Haphazard choices produce few rewards, as illustrated by performance after the second operation in Figure 4.

The proficiency of an animal's performance of the task can be measured by the ratio of trials to rewards. The most efficient strategy gives two rewards in every five trials, a ratio of 2.5 trials per reward, and less efficient strategies give a higher ratio. A Monte Carlo simulation of the task showed that with random choices there would be 16.3 trials per reward on average. We defined criterial performance as a ratio of 2.94 trials per reward, calculated on the basis that $85 \%$ of 2.94 is optimal performance (2.5), and the criterial performance can therefore be thought of as $85 \%$ of optimal. Training was administered in daily sessions, and the last reward earned in each session was the large food reward (see Apparatus). Trials in each session continued until a predetermined number of rewards had been earned. The number of rewards to be earned was usually 50 rewards, but this was reduced if an animal appeared reluctant to work in the early stages of training.

Preoperatively there was a program of introductory training in which simpler versions of the task were administered. Each animal began with only one pair of objects, and the value that the $\mathrm{P}$ choice accumulator had to reach to deliver a $\mathrm{P}$ reward or make available an $\mathrm{S}$ reward, the accumulator threshold that was set at four in the main task as described above, was initially set at two. When the animal achieved a ratio of 2.94 trials per reward in a single session, the accumulator threshold was then set to three, and when the animal again reached a ratio of 2.94 trials per reward, the accumulator threshold was finally increased to four, and training continued until the animal achieved a ratio of 2.94 trials per reward in each of two successive sessions. After reaching this criterion with one pair of objects, the same procedure was repeated with three further pairs one by one, but the introductory trials with an accumulator threshold of two or three were only one session, rather than being trained to a criterion, with the pairs after the first pair. Each of these further three pairs of objects was trained to the same criterion as the first pair, namely two successive days with 2.94 or fewer trials per reward, at an accumulator threshold of four. After criterion was reached with the fourth pair, the four pairs were presented concurrently in each session in random order; this was the main task as described above. Preoperative training in this main task continued until criterial performance was attained in each of two successive daily sessions.

Procedure: implementation of the strategy task before and after operation. On the completion of preoperative training in the strategy task, there was a period of $14 \mathrm{~d}$ without training, followed by a preoperative retention test. Subsequently, after each of the two surgical operations, there was a postoperative retention test. In all of these retention tests, daily sessions continued until a total of 300 rewards had been earned. In the preoperative retention test, there were 50 rewards per session, as in preoperative training. The same was true of those postoperative tests that showed no impairment, that is, after the first surgery for both groups and after the second surgery for group $\mathrm{TS}+\mathrm{AM}+\mathrm{FX}$. After the second surgery for group IT $\times$ FC, however, performance was so severely impaired that these animals were required to earn only 30 rewards in each session. The sessions at this retention test continued until 300 rewards had been earned in total, as in the previous retention tests, and thus, this retention test consisted of 10 sessions of 30 rewards.

Procedure: postoperative object-reward associative learning. After completing the final retention test of the strategy task, all the animals except
S5 were transferred to the associative learning task. (S5 was transferred to a different experiment, not described here.) The procedure was identical to that which was followed in our previous experiments, which tested the effects of the same lesions on the same task (Parker and Gaffan, 1998; Gaffan et al., 2001). On any trial, two objects were displayed on the left and right sides of the screen. Any such pair of objects constituted a reward-association learning problem, one object having been designated the correct (rewarded) one in the pair and the other the wrong one. The monkey chose one object by touching it, and both objects then disappeared. If the chosen object was the correct one, a food reward (190 mg) was dispensed. During the intertrial interval of $10 \mathrm{sec}$, any touch to the screen reset the interval. Sessions continued until 100 correct choices had been made, and the last correct choice was rewarded with the large food reward (see Apparatus). Objects were learned in sets of 10 problems (object pairs) concurrently. Each object in any new set was an object that the monkey had not encountered before. In successive runs of 10 trials, each problem was presented once. The order of problems within a 10-trial run was random. The animal continued daily sessions with a set of 10 until a criterion was met of $90 \%$ correct choices in the whole session (111 or fewer trials to earn 100 rewards), in sessions after the first session with that set, or of $90 \%$ correct choices after the first 10 trials, in the first session with that set. Learning ability was assessed by the number of errors committed, including those made in the criterial session, but excluding errors made during the first 10 trials with a new set of objects, when performance was necessarily at chance. The day after reaching criterion on one set, the animal began training with a new set. The animals learned three sets of 10 problems in this manner.

\section{RESULTS}

\section{Preoperative acquisition of the strategy task}

Table 2 shows results from individual monkeys in learning the strategy task with four pairs of objects acquired one after another (1-4) and subsequently with all four of those pairs combined (All). Each session of training continued until a certain number of rewards had been earned, and learning rate is therefore expressed as rewards to criterion, excluding the criterial sessions. The two groups that were subsequently assigned to receive different surgical operations (group IT $\times \mathrm{FC}$ and group $\mathrm{TS}+\mathrm{AM}+\mathrm{FX}$ ) learned the task preoperatively at a similar rate to each other, as is seen in the total number of rewards to criterion (Total). Furthermore, it can be seen that among the four successive object pairs (1-4), each was on average learned more quickly than the one before. The final column in Table 1 (First ratio) shows the trials per reward in a single session of training, the session when all four previously learned object pairs were combined together for the first time. It can be seen that all animals performed at a high level in this first session.

\section{Recovery from surgery}

Recovery from surgery was uneventful in all cases. The animals did not show any behavioral abnormalities in the home cages. 


\begin{tabular}{|c|c|c|c|}
\hline Monkey & Pre & Post1 & Post2 \\
\hline S1 & 3.0 & 3.4 & 8.6 \\
\hline S2 & 2.9 & 3.1 & 6.3 \\
\hline S3 & 2.9 & 3.1 & 9.3 \\
\hline $\mathrm{IT} \times \mathrm{FC}$ mean & 2.9 & 3.2 & 8.1 \\
\hline S4 & 2.9 & 3.1 & 3.2 \\
\hline S5 & 3.0 & 3.1 & 3.0 \\
\hline S6 & 3.0 & 3.3 & 3.3 \\
\hline $\mathrm{TS}+\mathrm{AM}+\mathrm{FX}$ mean & 3.0 & 3.2 & 3.2 \\
\hline
\end{tabular}

Pre, Performance in the preoperative retention test; Post1, performance after the first operation; Post2, performance after the second operation. See Table 1 for the sequence of surgical operations in each animal.

\section{Implementation of the strategy task before and after operation}

The preoperative retention test of the strategy task showed good retention in all individuals, as shown in the left column of Table 3 (Pre). Similarly, after the first surgical operation (Post1; see Table 1 for the sequence of surgical operations in each animal), performance remained good in both groups. However, after the second operation (Post2), performance was severely impaired in group IT $\times$ FC but not in group $\mathrm{TS}+\mathrm{AM}+\mathrm{FX}$. The group means are shown graphically in the left panel of Figure 3. Statistical analysis confirmed the effects seen. After the first operation there was a small but reliable deterioration of performance, by comparison with the preoperative retention test $\left(F_{(1,4)}=28.00 ; p=\right.$ 0.006), but the size of this effect was not different between the two groups $\left(F_{(1,4)}<1\right)$. The effect of the second operation, measured by comparison with performance after the first operation, differed markedly between the two surgical groups $\left(F_{(1,4)}=30.321\right.$; $p=0.005)$.

Trial-by-trial performance at each surgical stage is illustrated in Figure 4 from an individual animal in group IT $\times$ FC. It can be seen that performance in the preoperative retention test was proficient; in the illustrated preoperative session, there were three inappropriate sporadic choices and two failures to obtain a sporadic reward that was available, and the rest of the session was optimal. Similarly, after a unilateral frontal ablation in the first operation, performance remained proficient. However, after a crossed unilateral inferior temporal ablation in the second operation, the animal's choices became disorganized.

It can be seen in the final session in Figure 4 that after the second operation, this animal not only made sporadic choices when they were inappropriate but also failed to make sporadic choices when they were appropriate, that is, when they would have been rewarded. The data from all animals in the IT $\times$ FC group were analyzed to investigate the reliability of this effect. Figure 5 shows, for each of the three retention tests, the probability of a sporadic choice as a function of the number of preceding persistent choices. Optimal performance requires that a sporadic choice be made only after four persistent choices, the fourth of which is rewarded. Preoperatively and after the first surgery, choices were close to optimal, in that there was a low probability of sporadic choices intruding into the sequence pppP and a high probability of a sporadic choice after a rewarded $\mathrm{P}$ choice, when a sporadic reward was always available. After the second operation in this group, not only did the probability of making an inappropriate sporadic choice increase, but also the probability of making an

\section{Strategy implementation}

Associative learning

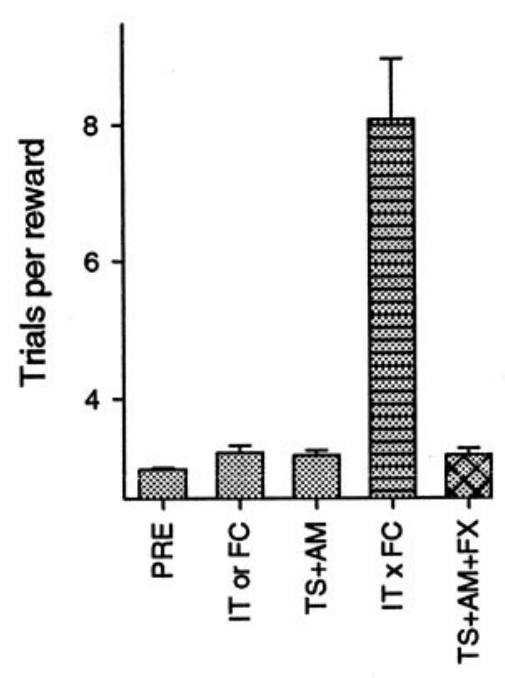

Groups

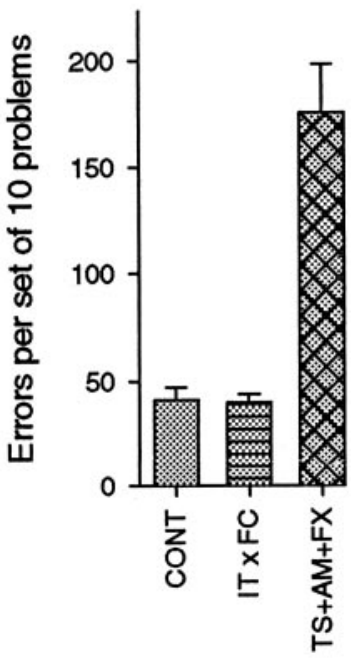

Groups
Figure 3. Summary of the ablation effects in both tasks. The left panel shows implementation of the preoperatively acquired strategy task. The origin of the vertical axis in this panel is 2.5 , which is optimal performance, and higher values indicate less proficient performance. $P R E$ shows the preoperative retention test. IT or $F C$ shows performance after the first operation in group IT $\times \mathrm{FC}$; see Tables 1 and 3 for details. $T S+A M$ shows performance after the first operation in group $\mathrm{TS}+\mathrm{AM}+\mathrm{FX} . I T \times F C$ shows performance after the second operation in group IT $\times$ FC. $T S+A M+F X$ shows performance after the second operation in group $\mathrm{TS}+\mathrm{AM}+\mathrm{FX}$. The right panel shows object-reward association learning. The data from the control group in the right panel (CONT) are from a group of normal animals, previously reported by Gaffan et al. (2001), performing exactly the same task, in the same apparatus, as the present newly reported animals. Group $I T \times F C$ in the right panel comprises both the present newly reported animals S1-S3 and also the animals performing the same task after the same lesions in the experiment by Parker and Gaffan (1998). Group $T S+A M+F X$ in the right panel comprises both the present newly reported animals S4 and S6 and also the animals performing the same task after the same lesions in the experiment by Gaffan et al. (2001). In each panel the error bars represent the SEMs. Data from individual monkeys are in Table 5.

appropriate sporadic choice decreased. The data from individual monkeys are presented in Table 4. Statistical analyses confirmed the reliability of the effects seen. One analysis examined choices after the sequences $\mathrm{p}$, pp, or ppp. Here, the probability of a sporadic choice showed a significant interaction of surgical stage with number of preceding persistent choices $\left(F_{(4,8)}=5.430\right.$; Huynh-Feldt $p=0.021$ ), as well as a significant main effect of surgical stage $\left(F_{(2,4)}=42.977\right.$; Huynh-Feldt $\left.p=0.009\right)$. The main effect of surgical stage confirms that sporadic choices were more frequent in these sequences after the second operation, and the interaction reflects the fact that preoperatively the animals were increasingly less likely to intrude a sporadic choice as the sequence pppP progressed trial by trial, whereas postoperatively there was no such change in probability of a sporadic choice. The preoperative behavior is rational because the cost of intruding a sporadic choice, in terms of the increase in the number of trials between two rewards, is greater after ppp than after $\mathrm{p}$, for example. A second analysis examined choices after the sequence pppP, when a sporadic choice was the best choice and would always be rewarded if made. Here, the probability of a sporadic choice declined after the second operation $\left(t_{(2)}=3.062 ; p=0.046\right.$, one-tailed). Thus, the 
End of pre-operative training: pppPSpppP SpppPS sppp: S SpppPSppp PSpppPSp

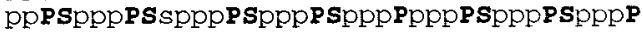
Sppp P Sppp P Spp PSpppPSpppPSpppPSpppPSpp pPSpppPSpppPpppPS

(50 rewards in 131 trials)

After unilateral frontal ablation: pppPS Sppp P Sppp P Sppp P SpppP SpppPSpppPSsp ppPSppp P Sppp P SpppP S Sppp P S sppp P S SpppPS sppp P Sppp PS sppp P S sppp PSppp $\mathbf{S}$ spspp sppp $\boldsymbol{P}$ pppPpppPS S S SpppPS spppPS S Sppp PSpppPS (50 rewards in 149 trials)

\begin{tabular}{|c|}
\hline 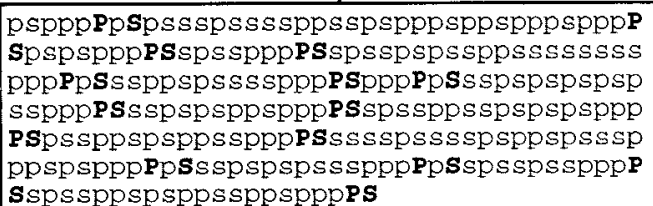 \\
\hline
\end{tabular}
( 30 rewards in 250 trials)

Figure 4. Three complete sessions by animal S1 in Group IT $\times$ FC. These are the final sessions in the three retention tests, which were a preoperative retention test, a postoperative retention test after the first surgery to remove frontal cortex unilaterally, and a final postoperative retention test after the second surgery to remove the contralateral inferior temporal cortex unilaterally. Each letter shows the individual monkey's choice on a single trial of the session. The letter $p$ or $P$ indicates the choice of a persistence-type object, and $s$ or $S$ indicates the choice of a sporadictype object. Lowercase letters indicate unrewarded choices, and uppercase letters indicate choices that produced a food reward.

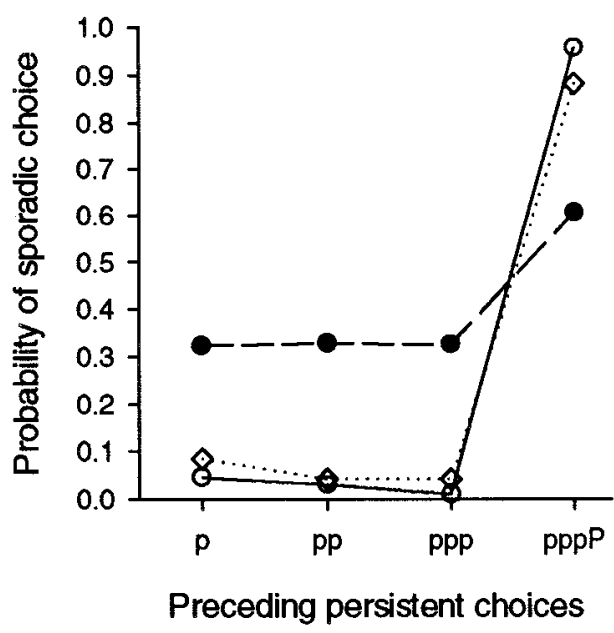

Figure 5. Probability of a sporadic choice as a function of preceding choices, at each of the three surgical stages in Group IT $\times$ FC (monkeys $\mathrm{S} 1-\mathrm{S} 3$ ). The data from individual monkeys are given in Table 4. $p=$ an unrewarded persistent choice; $P=$ a rewarded persistent choice (as in Fig. 4). $\bigcirc$ indicates preoperative performance; $\diamond$ indicates performance after the first operation; indicates performance after the second operation.

impairment was not only a disinhibition of sporadic choices, but also a failure to produce sporadic choices when they were appropriate. It should also be noted, however, that although the overall performance after the second operation was severely impaired in the ways that have been described, it was not at chance, which would be 16.3 trials per reward (see Materials and Methods).

A final analysis examined performance session by session after the second operation in group IT $\times$ FC to determine whether they showed signs of recovering performance with postoperative
Table 4. Probability of a sporadic choice in the strategy task in monkeys in group IT $\times$ FC

\begin{tabular}{lllll} 
& \multicolumn{4}{l}{ Preceding choices } \\
\cline { 2 - 5 } Monkey & $\mathrm{p}$ & $\mathrm{pp}$ & $\mathrm{ppp}$ & $\mathrm{pppP}$ \\
\hline PRE & & & & \\
S1 & 0.061 & 0.028 & 0.019 & 0.959 \\
S2 & 0.036 & 0.031 & 0.013 & 0.974 \\
S3 & 0.044 & 0.031 & 0 & 0.934 \\
Mean & 0.047 & 0.030 & 0.011 & 0.956 \\
POST 1 (IT or FC) & & & & \\
S1 & 0.086 & 0.047 & 0.037 & 0.941 \\
S2 & 0.049 & 0.023 & 0.048 & 0.861 \\
S3 & 0.120 & 0.060 & 0.040 & 0.848 \\
Mean & 0.085 & 0.043 & 0.042 & 0.883 \\
POST 2 (IT $\times$ FC) & & & & \\
S1 & 0.393 & 0.395 & 0.401 & 0.814 \\
S2 & 0.227 & 0.249 & 0.238 & 0.421 \\
S3 & 0.353 & 0.343 & 0.340 & 0.583 \\
Mean & 0.324 & 0.329 & 0.326 & 0.606 \\
\hline P A & & & &
\end{tabular}

p, An unrewarded persistent choice; $\mathrm{P}$, a rewarded persistent choice (see Figs. 4 and 5).

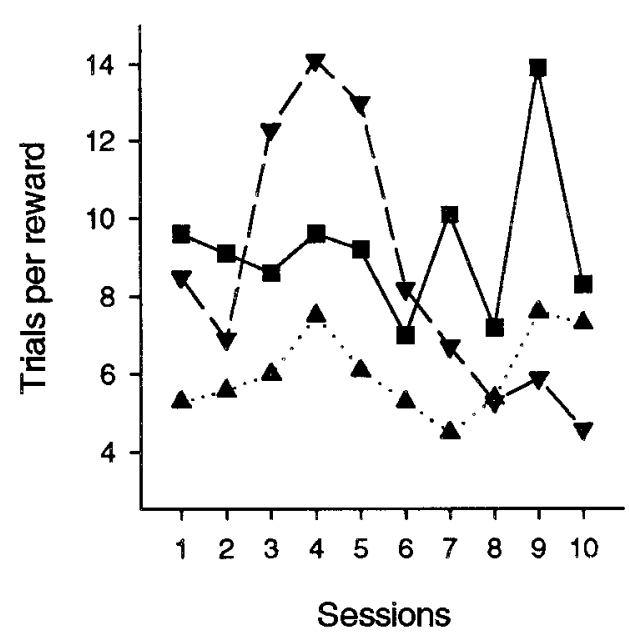

Figure 6. Trials per reward in each of the 10 sessions of the final postoperative retention test in Group IT $\times$ FC (monkeys S1-S3). $\mathbf{\square}, \mathrm{S} 1$; $\boldsymbol{\Delta}, \mathrm{S} 2 ; \boldsymbol{\nabla}, \mathrm{S} 3$.

practice. Figure 6 shows that, for each of the three individual monkeys in this group, performance did not improve over the 10 sessions of the retention test. There was no significant correlation of performance with session number in any of the animals or in the group as a whole (the greatest absolute correlation coefficient between trials per reward and session number in the individual monkeys was $r_{(8)}=-0.562, p=0.091$, and the same correlation coefficient for the group mean performance was $r_{(8)}=-0.301$, $p=0.398)$.

\section{Postoperative object-reward associative learning}

Figure 3, right panel, shows errors to criterion during objectreward association learning. Results from the individual animals are given in Table 5. The data from the control group in Figure $3(C O N T)$ are from a previously reported group of eight normal Rhesus and Cynomolgus monkeys, performing exactly the same task, in the same apparatus, as the present newly reported animals [these were animals A1-R1 in Table 4 in Gaffan et al. (2001)]. 


\begin{tabular}{lcc}
\hline $\begin{array}{l}\text { Table 5. Errors to criterion per set of } \mathbf{1 0} \text { problems in object-reward } \\
\text { association learning }\end{array}$ & \\
Group & Monkey & Errors \\
\hline CONT & PP1 & 11.8 \\
& PP2 & 55.6 \\
& PP3 & 31.8 \\
& PP4 & 33.8 \\
& PP5 & 39.4 \\
& PP6 & 39.8 \\
& PP7 & 57.6 \\
& PP8 & 60.2 \\
IT $\times$ FC & S1 & 45.3 \\
& S2 & 33.3 \\
& S3 & 28.0 \\
PS9 & 41.0 \\
PM+FX & PP10 & 52.0 \\
& S4 & 193.3 \\
& S6 & 172.7 \\
& PP11 & 67.0 \\
& PP12 & 224.0 \\
& PP13 & 195.0 \\
& PP14 & 202.3 \\
& & \\
& &
\end{tabular}

Monkeys S1-S6 are the present newly reported monkeys; PP1-14 are previously published monkeys performing the same task, as further specified in Results. The group means are shown in Figure 3.

Group IT $\times$ FC in Figure 3, right panel, comprises the present newly reported animals $\mathrm{S} 1-\mathrm{S} 3$ and also the two animals that performed the same task after the same lesions in an earlier experiment [these were animals OP5 and OP6 in Table 3 in Parker and Gaffan (1998)]. The errors to criterion per set of 10 problems that were made by the new animals S1-S3 were similar to those made by the two animals with the same lesions in the earlier experiment (Table 5). Group TS $+\mathrm{AM}+\mathrm{FX}$ in Figure 3, right panel, comprises the present newly reported animals $\mathrm{S} 4$ and S6 (S5 did not perform this task; see Materials and Methods) and also the four animals that performed the same task after the same lesions in an earlier experiment [animals A3, B3, C1, and O2 in Table 4 in Gaffan et al. (2001)]. The errors to criterion per set of 10 problems that were made by the new animals S4 and S6 were similar to those made by the four animals with the same lesions in the earlier experiment (Table 5). ANOVA showed that the three groups in Figure 3 differed significantly in their proficiency of associative learning $\left(F_{(2,16)}=33.695, p=0.000\right)$. A designed comparison using the pooled error term showed that learning in group $\mathrm{TS}+\mathrm{AM}+\mathrm{FX}$ was significantly less proficient than in group IT $\times \mathrm{FC}\left(t_{(16)}=6.377, p=0.000\right)$.

\section{DISCUSSION}

The most important aspect of the preoperative data in the strategy task was the good performance that we observed when four pairs of objects, which had been learned separately, were first put together and tested concurrently. The ratio of trials to rewards reflects proficiency in the task, optimal performance producing a ratio of 2.5 and chance performance a ratio of 16.3. The average of 3.03 in the first session with the four separately learned pairs put together for the first time (Table 2, First ratio) therefore represents good performance. This shows that the animals had not learned to use specific objects as conditional discrimination cues in learning the four pairs separately. In principle, an animal could have learned, for example, that in the first pair a reward for choosing object 1 is an instruction cue to switch to object 2, and vice versa. If so, however, there would have been no transfer when all four pairs were put together and now a reward for object 1 is followed by a choice between some different pair of objects, say 7 and 8 , which have never been trained with objects 1 and 2 . The good transfer to concurrent testing indicates that the animals had learned a more abstract rule, linking each object to one or other of the two available strategies for obtaining reward. This abstract rule, linking objects to strategies, is similar to some aspects of human cognitive capacity that are dependent on frontal function, including planning, the sequential organization of actions, and the application of strategies (Luria, 1966; Milner, 1982; Shallice, 1982; Duncan, 1986; Owen et al., 1990; Shallice and Burgess, 1991). In these human tasks, like the present strategy task and unlike previous conditional learning tasks with monkeys, there is frequently no discrete conditional cue that signals the appropriate action.

After crossed unilateral removals of frontal and inferior temporal cortex, in group IT $\times \mathrm{FC}$, the impairment in implementing the preoperatively learned strategy task was severe (Fig. 3) and stable (Fig. 6). Furthermore, the impairment in this task was not simply a failure to inhibit sporadic choices. Not only did the probability of an inappropriate sporadic choice increase postoperatively, but also the probability of an appropriate sporadic choice decreased (Fig. 5). The result was a general disorganization of choices (Fig. 4). At the same time, these animals were quite unimpaired in learning object-reward associations (Fig. 3, right panel), confirming previous results (Parker and Gaffan, 1998). These results, taken together, strengthen the existing evidence (for review, see Gaffan, 2002) that the prefrontal cortex is involved in learning and retrieving any kind of information that is not adequately processed in other, more specialized cortical areas. This includes, as the present results indicate, the retrieval of abstract rules.

The opposite pattern of results was produced by bilateral section of anterior temporal stem, amygdala, and fornix, in group $\mathrm{TS}+\mathrm{AM}+\mathrm{FX}$. Confirming previous results, these animals were severely impaired in object-reward associative learning (Fig. 3, right panel). The new and striking finding from this group, however, was that they implemented the preoperatively learned strategy task at an almost normal level of proficiency (Fig. 3, left panel). This result shows that the severe and general memory impairment shown by these animals in new postoperative learning (Gaffan et al., 2001) is not attributable to temporal-frontal disconnection. It also adds powerfully to the evidence (Easton and Gaffan, 2000; Gaffan et al., 2001) that these animals' impairment in acquiring new memories is much more severe than their impairment in retrieving memories that were acquired preoperatively.

There are several possible routes by which frontal cortex and inferior temporal cortex might communicate in the implementation of a strategy. Peripheral routes of interaction (Eacott and Gaffan, 1989) and interhemispheric routes, through the forebrain commissures or indirectly through subcortical structures that communicate across the midline, are clearly not sufficient for normal performance, in the light of the impairment seen in the animals with crossed unilateral removals. Furthermore, the normal performance of the strategy task by the animals with section of temporal stem, amygdala, and fornix (Fig. 3) shows that several possible routes of within-hemisphere temporal-frontal communication are not necessary for normal performance of the task. One such route is the monosynaptic, corticocortical route of 
interaction between frontal and inferior temporal cortex in the uncinate fascicle (Ungerleider et al., 1989), which is cut as part of the anterior temporal stem section in these animals. The present study reinforces the conclusion from other experiments that this monosynaptic route is necessary only for a small subset of tasks, namely conditional discriminations with a visual instruction cue (Eacott and Gaffan, 1992; Gaffan and Eacott, 1995a,b; Gutnikov et al., 1997). Another possible route of within-hemisphere temporal-frontal communication is mediated by the basal forebrain, through frontal influence on the basal forebrain and basal forebrain influence on the temporal lobe cortex (Mesulam and Mufson, 1984; Ongur et al., 1998; Rempel-Clower and Barbas, 1998). Like the monosynaptic route, this route is also ruled out as necessary for the performance of the strategy task by the unimpaired performance of the animals with section of fornix, amygdala, and temporal stem.

Discussing the negative effects of uncinate fascicle section, Gaffan and Eacott (1995b) suggested that temporal and frontal cortex could interact within each hemisphere within the corpus striatum, to which they both project. Wise et al. (1996) proposed a detailed model in which the corpus striatum potentiates behavioral rules. These suggestions offer one possible route through which temporal cortex and frontal cortex might interact in implementing the strategy task. However, parsimony argues against this proposal. In simple visual association learning for reward, several lines of evidence show that visual information can be retrieved from inferior temporal cortex, and used to select the choice of the appropriate object, by some other route than by the projection from inferior temporal cortex to the corpus striatum. First, electrophysiological recordings from this region, namely the part of the caudateputamen that receives the projection from inferior temporal cortex, show that in monkeys learning visual object-reward associations, the activity of the cells does not encode information about the object-reward associations or about the animals' choices (Brown et al., 1995). Second, the output from the basal ganglia is ultimately, via the thalamus, primarily to the frontal cortex itself (for review, see Wise et al., 1996), and therefore visual choices in visual reward-association learning, which is unimpaired by crossed unilateral lesions of frontal and temporal cortex, cannot be mediated by this route. Third, if a large area of nonvisual cortex is removed in the monkey, the animal can no longer make visual choices of any kind, although the basal ganglia and the visual cortex including inferior temporal cortex, are both intact (Nakamura and Mishkin, 1986). These three lines of evidence suggest that in object-reward association memory, the crucial output of inferior temporal cortex is to cortex (as further specified below) and not to the corpus striatum. However, if that transcortical output is sufficient and necessary for performance of visual object-reward association tasks, it is parsimonious to suppose that the same transcortical output is also involved in other more complex visual tasks, including conditional discriminations and the present strategy task.

This hypothesis is illustrated in Figure 7. The posterior cortical areas referred to in Figure 7 include parietal and prestriate cortical areas, which receive projections from both frontal cortex and inferior temporal cortex. We assume that these posterior areas can represent differentially the implementation of the two strategies for pursuit of food reward, persistent and sporadic, because those two strategies differ in the pattern of eye movements and hand movements that the animal makes. Frontal input to these posterior cortical areas therefore could selectively activate the representation of the currently active strategy, and reciprocal temporal connections with these posterior cortical areas
Strategy implementation

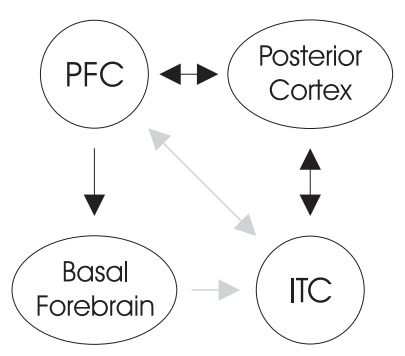

Associative learning

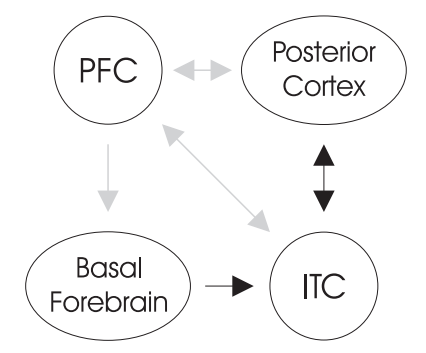

Figure 7. Proposed routes of interaction between inferior temporal cortex (ITC) and prefrontal cortex (PFC) in the two present tasks. For each task, heavy lines and arrows indicate pathways that are necessary, and light lines and arrows indicate pathways that exist but are not necessary for that task.

could then activate the representations of the appropriate objects for that strategy. A similar hypothesis of temporal interaction with posterior cortex in memory retrieval has been put forward to explain visual neglect (Gaffan and Hornak, 1997). These transcortical associative networks are created, as our previous results have indicated (Easton and Gaffan, 2000, 2001; Easton et al., 2001, 2002), by the influence of the basal forebrain on cortical association formation. Once created, however, they do not need basal forebrain input to function normally in implementation of the strategy or action that has been learned.

\section{REFERENCES}

Brown VJ, Desimone R, Mishkin M (1995) Responses of cells in the tail of the caudate nucleus during visual discrimination learning. J Neurophysiol 74:1083-1094.

Corkin S, Amaral DG, Gonzalez RG, Johnson KA, Hyman BT (1997) H. M.'s medial temporal lobe lesion: findings from magnetic resonance imaging. J Neurosci 17:3964-3979.

Duncan J (1986) Disorganization of behaviour after frontal lobe damage. Cognit Neuropsychol 3:271-290.

Eacott MJ, Gaffan D (1989) Reaching to a rewarded visual stimulus: interhemispheric conflict and hand use in monkeys with forebrain commissurotomy. Brain 112:1215-1230.

Eacott MJ, Gaffan D (1992) Inferotemporal-frontal disconnection: the uncinate fascicle and visual associative learning in monkeys. Eur J Neurosci 4:1320-1332.

Easton A, Gaffan D (2000) Comparison of perirhinal cortex ablation and crossed unilateral lesions of the medial forebrain bundle from the inferior temporal cortex in the rhesus monkey: effects on learning and retrieval. Behav Neurosci 114:1041-1057.

Easton A, Gaffan D (2001) Crossed unilateral lesions of the medial forebrain bundle and either inferior temporal or frontal cortex impair object-reward association learning in rhesus monkeys. Neuropsychologia 39:71-82.

Easton A, Parker A, Gaffan D (2001) Crossed unilateral lesions of medial forebrain bundle and either inferior temporal or frontal cortex impair object recognition memory in Rhesus monkeys. Behav Brain Res 121:1-10.

Easton A, Ridley RM, Baker HF, Gaffan D (2002) Unilateral lesions of the cholinergic basal forebrain and fornix in one hemisphere and inferior temporal cortex in the opposite hemisphere produce severe learning impairments in rhesus monkeys. Cereb Cortex 12:729-736.

Gaffan D (2002) Against memory systems. Philos Trans R Soc Lond B Biol Sci, in press.

Gaffan D, Eacott MJ (1995a) Uncinate fascicle section leaves delayed matching-to-sample intact, both with large and small stimulus sets. Exp Brain Res 105:175-180.

Gaffan D, Eacott MJ (1995b) Visual learning for an auditory secondary reinforcer is intact after uncinate fascicle section: indirect evidence for the involvement of the corpus striatum. Eur J Neurosci 7:1866-1871.

Gaffan D, Harrison S (1988) Inferotemporal-frontal disconnection and fornix transection in visuomotor conditional learning by monkeys. Behav Brain Res 31:149-163.

Gaffan D, Harrison S (1991) Auditory-visual associations, hemispheric specialization, and temporal-frontal interaction in the Rhesus monkey. Brain 114:2133-2144. 
Gaffan D, Hornak J (1997) Visual neglect in the monkey: representation and disconnection. Brain 120:1647-1657.

Gaffan D, Saunders RC, Gaffan EA, Harrison S, Shields C, Owen MJ (1984) Effects of fornix transection upon associative memory in monkeys: role of the hippocampus in learned action. Q J Exp Psychol 36B: $173-221$

Gaffan D, Parker A, Easton A (2001) Dense amnesia in the monkey after transection of fornix, amygdala and anterior temporal stem. Neuropsychologia 39:51-70.

Gutnikov SA, Ma Y, Gaffan D (1997) Temporo-frontal disconnection impairs visual-visual paired association learning but not configural learning in macaca monkeys. Eur J Neurosci 9:1524-1529.

Luria AR (1966) Higher cortical functions in man. London: Tavistock.

Maclean CJ, Gaffan D, Baker HF, Ridley RM (2001) Visual discrimination learning impairments produced by combined transections of the anterior temporal stem, amygdala and fornix in marmoset monkeys. Brain Res 888:34-50.

Mesulam M-M, Mufson EJ (1984) Neural inputs into the nucleus basalis of the substantia innominata (Ch4) in the rhesus monkey brain. Brain 107:253-274.

Milner B (1982) Some cognitive effects of frontal lobe lesions in man. Philos Trans R Soc Lond B Biol Sci 298:211-266.

Nakamura RK, Mishkin M (1986) Chronic "blindness" following lesions of nonvisual cortex in monkey. Exp Brain Res 63:173-184.
Ongur D, An X, Price JL (1998) Prefrontal cortical projections to the hypothalamus in macaque monkeys. J Comp Neurol 401:480-505.

Owen AM, Downes JJ, Sahakian BJ, Polkey CE, Robbins TW (1990) Planning and spatial working memory following frontal lobe lesions in man. Neuropsychologia 28:1021-1034.

Parker A, Gaffan D (1998) Memory after frontal-temporal disconnection in monkeys: conditional and nonconditional tasks, unilateral and bilateral frontal lesions. Neuropsychologia 36:259-271.

Rempel-Clower NL, Barbas H (1998) Topographic organization of connections between the hypothalamus and prefrontal cortex in the rhesus monkey. J Comp Neurol 398:393-419.

Selden NR, Gitelman DR, Salamon-Murayama N, Parrish TB, Mesulam MM (1998) Trajectories of cholinergic pathways within the cerebral hemispheres of the human brain. Brain 121:2249-2257.

Shallice T (1982) Specific impairments in planning. Philos Trans R Soc Lond B Biol Sci 298:199-209.

Shallice T, Burgess PW (1991) Deficits in strategy application following frontal-lobe damage in man. Brain 114:727-741.

Ungerleider LG, Gaffan D, Pelak VS (1989) Projections from inferior temporal cortex to prefrontal cortex via the uncinate fascicle in Rhesus monkeys. Exp Brain Res 76:473-484.

Wise SP, Murray EA, Gerfen CR (1996) The frontal cortex-basal ganglia system in primates. Crit Rev Neurobiol 10:317-356. 